\title{
Current challenges in cell wall biology in the cereals and grasses
}

\author{
Rachel A. Burton and Geoffrey B. Fincher* \\ Australian Research Council Centre of Excellence in Plant Cell Walls, School of Agriculture, Food and Wine, University of Adelaide, Glen Osmond, SA, Australia
}

\section{Edited by:}

Jose Manuel Estevez, University of Buenos Aires and Consejo Nacional de Investigaciones Científicas y

Técnicas, Argentina

\section{Reviewed by:}

Thorsten Hamann, Imperial College London, UK

Bjoern Usadel, Max Planck Institute of Molecular Plant Physiology, Germany

\section{${ }^{*}$ Correspondence:}

Geoffrey B. Fincher, Australian

Research Council Centre of

Excellence in Plant Cell Walls, School

of Agriculture, Food and Wine,

University of Adelaide, Waite Campus,

Glen Osmond, SA 5064, Australia.

e-mail:geoff.fincher@adelaide.edu.au
Plant cell walls consist predominantly of polysaccharides and lignin. There has been a surge of research activity in plant cell wall biology in recent years, in two key areas. Firstly, in the area of human health it is now recognized that cell wall polysaccharides are key components of dietary fiber, which carries significant health benefits. Secondly, plant cell walls are major constituents of lignocellulosic residues that are being developed as renewable sources of liquid transport biofuels. In both areas, the cell walls of the Poaceae, which include the cereals and grasses, are particularly important. The non-cellulosic wall polysaccharides of the Poaceae differ in comparison with those of other vascular plants, insofar as they contain relatively high levels of heteroxylans as "core" polysaccharide constituents and relatively smaller amounts of heteromannans, pectic polysaccharides, and xyloglucans. Certain grasses and cereals walls also contain $(1,3 ; 1,4)-\beta$-glucans, which are not widely distributed outside the Poaceae. Although some genes involved in cellulose, heteroxylan, and $(1,3 ; 1,4)-\beta$-glucan synthesis have been identified, mechanisms that control expression of the genes are not well defined. Here we review current knowledge of cell wall biology in plants and highlight emerging technologies that are providing new and exciting insights into the most challenging questions related to the synthesis, re-modeling and degradation of wall polysaccharides.

Keywords: biofuels, cell biology, gene identification, glycosyl transferases, human health, polysaccharide biosynthesis, regulation

\section{INTRODUCTION}

Two major drivers have contributed to dramatic increases in international research activities in the area of plant cell wall biology in recent years. Firstly, the enormous potential for crop residues and specialty plant bioenergy species as a large scale source of renewable liquid transport fuels is attracting both private and public sector research and development investment in the field (McLaren, 2005). Cell wall constituents represent the most important energy source in these plant materials. The second driver is the recognition that many of the world's most widespread diseases, such as type II diabetes, colorectal cancer, cardiovascular, and certain inflammatory diseases, can be alleviated to varying extents by increasing the fiber content of human diets (Jacobs and Gallaher, 2004; Jemal et al., 2005; Topping, 2007; Collins et al., 2010). Notably, cell wall constituents represent a major component of dietary fiber in human societies around the world. Plant cell walls consist predominantly of polysaccharides and lignin. While the biochemical pathways of lignin synthesis are now well characterized and new genetic technologies have been successfully used to manipulate both the total amount and the chemical structure of lignin in plants, our understanding of the genes and enzymes involved in cell wall polysaccharide synthesis is still somewhat limited; we will focus on the biology of wall polysaccharides in this short review. It is estimated that hundreds of genes participate in cell wall polysaccharide metabolism in plants, but the functions of a relatively small proportion of these have been unequivocally assigned.
Here we will focus on current challenges in cell wall biology in the Poaceae, because these species are particularly important in human societies. Thus, grains from rice, wheat, sorghum, millet, and barley represent a large proportion of daily caloric intake in human diets and sugar cane is widely grown as a source of sucrose. Numerous fodder and forage grass species are used for the production of domestic animals and maize is also used for feeding livestock. Cereal crop residues are potential sources of lignocellulose for the production of bioethanol and other biofuels (McLaren, 2005) and perennial grasses, including switchgrass and Miscanthus, are being developed as specialist bioenergy crops, particularly in North America and Europe.

\section{COMPOSITION OF CELL WALLS IN THE GRASSES}

Cell walls in the Poaceae are markedly different in composition compared with those from other monocots and from dicots (Gibeaut and Carpita, 1993; Trethewey et al., 2005). These characteristic differences are manifest in the non-cellulosic polysaccharide components. In dicots and non-commelinoid monocotyledons, the major non-cellulosic polysaccharides of primary walls are xyloglucans and a range of pectic polysaccharides, with lower levels of heteroxylans and heteromannans. In contrast, primary walls of grasses have much lower levels of xyloglucans and pectins, which are replaced by higher amounts of heteroxylans and, in some cases, with $(1,3 ; 1,4)-\beta$-glucans.

The heteroxylans of the grasses consist of a $(1,4)$-linked backbone of $\beta$-D-xylopyranosyl (Xylp) residues, to which 


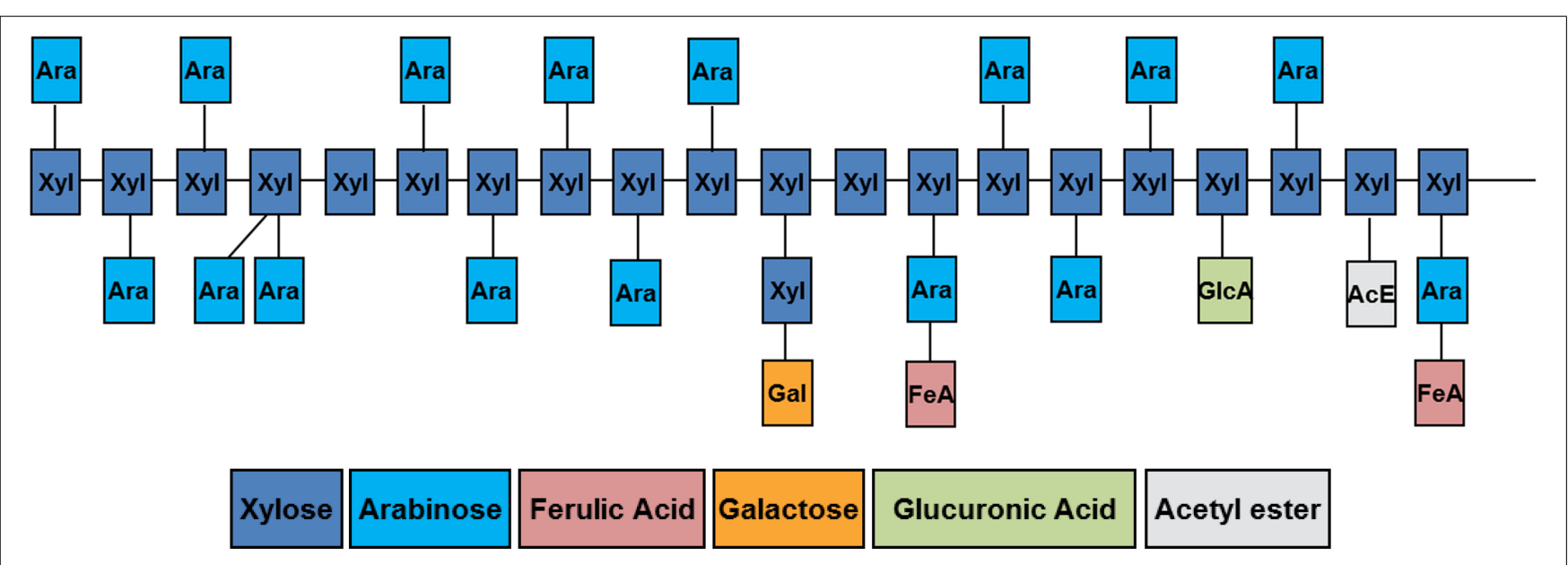

FIGURE 1 | Diagrammatical representation of structural features found in heteroxylans of the grasses. Details of the linkage types involved in the polysaccharides are outlined in the text. Figure prepared by Dr Hunter Laidlaw.

is appended single $\alpha$-L-arabinofuranosyl (Araf), single $\alpha-D$ glucuronopyranosyl (GlcpA) residues, or the 4-O-methyl ethers of GlcpA residues (Izydorczyk and Biliaderis, 1994; Fincher and Stone, 2004). In some cases oligosaccharide substituents such as $\beta$-D-Xyl $p$ - $(1,2)$-L-Araf- $(1-$ and $\beta$-D-Gal $p(1,4)-\beta$-D-Xyl $p$ - $(1,2)$ L-Araf-(1- are also detected in heteroxylans (Fincher and Stone, 2004). The Araf residues are usually linked to the $\mathrm{C}(\mathrm{O}) 3$ position of the Xylp residues, but on occasions are found on $\mathrm{C}(\mathrm{O}) 2$ or on both of these carbon atoms. The GlcpA residues are usually linked to the $\mathrm{C}(\mathrm{O}) 2$ atom of the $\mathrm{Xyl} p$ residues. The structural features of plant heteroxylans are summarized in Figure $\mathbf{1}$. The number and distribution of substituents along the $(1,4)-\beta$ xylan backbone vary between and within species, and are major determinants of the physicochemical properties of the heteroxylans (Ebringerová, 2005). In the heteroxylans from the starchy endosperm of grasses, the major substituents are Araf residues; GlcpA residues are less common. If the $(1,4)-\beta$-xylan backbone is heavily substituted with Araf residues, the polysaccharide will be more soluble, because the Araf residues sterically inhibit intermolecular alignment of individual molecules and prevent aggregation and precipitation. Physical alignment will also occur between arabinoxylans and cellulose, in regions where lengths of the $(1,4)-\beta$-xylan backbone are unsubstituted. The solubility of the arabinoxylan can be predicted from the Xyl:Ara ratio of the polysaccharide, at least in general terms. For example, less substituted, less soluble arabinoxylans are found in the pericarptesta of cereal bran and in other secondary cell wall material (Fincher and Stone, 2004).

A proportion of the Araf residues of arabinoxylans from the grasses are substituted at $\mathrm{C}(\mathrm{O}) 5$ with hydroxycinnamic acids; ferulic acid is the common hydroxycinnamic acid, but $p$-coumaric acid is also found. Ferulic acid residues account for up to $1.8 \%$ (w/w) of wheat aleurone walls (Bacic and Stone, 1981) and 0.04\% of barley starchy endosperm walls (Fincher, 1975). Oxidative coupling of feruloyl residues on adjacent arabinoxylan chains is believed to allow covalent cross-linking of polysaccharide chains in the wall.

The other major non-cellulosic polysaccharides of cell walls in the grasses are the $(1,3 ; 1,4)-\beta$-glucans. These consist of unbranched, unsubstituted chains of $\beta$-D-glucopyranosyl residues, joined by $(1,3)$ - and $(1,4)$-linkages. In the majority of cases in cereal grains, blocks of two or three adjacent $(1,4)$-linkages are separated by single $(1,3)$-linkages, although blocks of up to 10 or 12 adjacent $(1,4)$-linkages occur at relatively low frequencies (Woodward etal., 1983). Thus, there is no repeating unit in the $(1,3 ; 1,4)-\beta$-glucans from cereal grains. However, the $(1,3)$ - and $(1,4)$-linkages are not arranged at random, because contiguous (1,3)-linkages are generally not detected (Staudte et al., 1983). Given that there are usually $25-30 \%(1,3)-\beta$-glucosyl residues in the polysaccharide, one would expect that if the $(1,3)-\beta$-glucosyl and $(1,4)-\beta$-glucosyl residues were arranged at random significant levels of contiguous $(1,3)-\beta$-glucosyl residues would be present. This is not the case.

Another way of viewing $(1,3 ; 1,4)-\beta$-glucans structure is that the polysaccharide is composed predominantly of cellotriosyl (with a degree of polymerization of DP3) and cellotetraosyl (DP4) residues linked by single $(1,3)-\beta$-linkages, as shown below:

\section{.. G 3 G 4 G 4 G 34 G 4 G 4 G 3 G 4 G 4 G 3 G .. \\ cellotriosyl \\ (DP3)}

Markov chain analyses allowed Staudte et al. (1983) to demonstrate that the blocks of two or three adjacent $(1,4)$-linked glucosyl residues are arranged at random, which indicated that the $(1,3)$ linked glucosyl residues would be irregularly spaced along the polysaccharide chain. The irregularly spaced $(1,3)-\beta$-glucosyl residues will introduce irregularly spaced molecular kinks along what would otherwise be a linear, ribbon-like $(1,4)-\beta$-glucan, or cellulosic backbone. Again the fine structure of the polysaccharide, expressed this time as the DP3:DP4 ratio, will allow us to predict its physicochemical properties (Lazaridou and Biliaderis, 2004; Burton etal., 2010). When the DP3:DP4 ratios of cereal 
Table 1 | Levels of $(1,3 ; 1,4)-\beta$-glucans in grains of various grass species.

\begin{tabular}{|c|c|c|c|c|}
\hline Species & $\begin{array}{l}(1,3 ; 1,4)-\beta \text {-glucan } \\
(\% \mathrm{w} / \mathrm{w})\end{array}$ & $\begin{array}{l}\text { DP3:DP4 } \\
\text { ratio }\end{array}$ & $\begin{array}{l}\text { Solubility } \\
\text { in water (\%) }\end{array}$ & $\begin{array}{l}\text { Starch } \\
(\% \mathrm{w} / \mathrm{w})\end{array}$ \\
\hline Oats & $6-8$ & $1.5-2.3: 1$ & $\sim 80$ & $\sim 60$ \\
\hline Barley & $4-10$ & $2.6: 1$ & $\sim 20$ & $\sim 57$ \\
\hline Wheat & 1 & $3.2: 1$ & 0 & $\sim 57$ \\
\hline Brachypodium & 40 & $5.9: 1$ & Insoluble & $<10$ \\
\hline Rice & $<0.06$ & $1: 1.4$ & $?$ & 80 \\
\hline Maize & Trace & $2.5: 1$ & $?$ & $70-80$ \\
\hline Sorghum & $0.07-0.2$ & 2.8:1 & $?$ & $70-80$ \\
\hline $\begin{array}{l}\text { Transgenic barley } \\
\text { (AsGlo:Cs/F6) }\end{array}$ & 7.8 & 2.1 & $?$ & 44 \\
\hline $\begin{array}{l}\text { Transgenic barley } \\
\text { (AsGlo:Cs/F4) }\end{array}$ & 6.0 & 2.8:1 & $?$ & $?$ \\
\hline
\end{tabular}

Only trace amounts can be detected in rice, maize, and sorghum grain, but in Brachypodium $(1,3 ; 1,4)-\beta$-glucans constitute up to $40 \%(w / W)$ of the grain. The link between DP3:DP4 ratios and polysaccharide solubility can also be seen. At the relatively low ratios observed in the oat grain $(1,3 ; 1,4)-\beta$-glucans, the polysaccharide is more soluble than it is in the other grains, where higher DP3:DP4 ratios are observed. The values shown for the barley AsGlo:CsIF lines were obtained from grain of transgenic barley transformed with the CsIF genes as described by Burton etal. (2011).

$(1,3 ; 1,4)-\beta$-glucans are in the range of about $2.0-3.0$, at least some of the polysaccharide will be soluble in aqueous media. As the ratios move further from these values, in either direction, the polysaccharide becomes increasingly comprised of DP3 or DP4 units and hence individual molecules become more regular in shape and can more easily align over extended regions (Burton et al., 2010). As a result, the $(1,3 ; 1,4)$ - $\beta$-glucans will become less soluble as the DP3:DP4 ratio deviates from values of 1:1 although this may be influenced by physical or chemical interactions with other polymers found in the cereal grain or in downstream industrial processes. Selected examples showing the different fine structures of $(1,3 ; 1,4)-\beta$-glucans from grasses are shown in Table 1. It should be noted that the $(1,3 ; 1,4)-\beta$-glucans found in isolated cases in lower plants of the monilophyte group and in fungal walls have quite different structures, insofar as either the DP3 or DP4 unit predominates and the polysaccharide can therefore be viewed as having a repeating DP3 or DP4 unit structure (Pettolino et al., 2009; Xue and Fry, 2012). Perhaps it is the ability of the grasses to synthesize a non-repeating $(1,3 ; 1,4)$ - $\beta$-glucan comprising DP3, DP4, and longer cellodextrin units that has led to the more widespread adoption of the polysaccharide in the grasses.

\section{CHALLENGE I: HETEROXYLAN BIOSYNTHESIS; WHICH GENES AND HOW?}

The "core" non-cellulosic polysaccharides of cell walls in the grasses are heteroxylans, also found in dicot walls but in much lower abundance. Structurally, heteroxylans have features that are similar to xyloglucans, which are widely distributed as a major non-cellulosic component of dicot walls. In both cases, aggregation of the polysaccharide chains into insoluble or fibrillar material is sterically inhibited through the addition of monosaccharide or oligosaccharide substituents to main chains that are both extended, highly asymmetrical $(1,4)-\beta$-glycans. The types of substituents, the degree of substitution and distribution patterns along the main chain vary both between species and cells within a single plant.

Much of the initial work on the identification of genes involved in heteroxylan synthesis was focused on analyses of Arabidopsis mutant lines and transcript profiling. These studies implicated genes from the GT8, GT43, GT47, and GT61 families (Brown et al., 2007, 2009; Mitchell et al., 2007; Pena et al., 2007; Persson et al., 2007; Oikawa et al., 2010). However, these experiments were plagued with interpretative difficulties imposed by the large gene families, compensation and pleiotropic effects in transgenic lines during proof-of-function tests, and the difficulties associated with developing reliable biochemical assays of expressed enzymes. Mitchell et al. (2007) and Pellny et al. (2012) used comparative bioinformatics analyses to find candidate genes and concluded that genes in the GT43 and GT47 families might encode backbone $(1,4)-\beta$-xylan synthases, genes in the GT61 family might encode xylan $(1,2)-\alpha$ - or $(1,3)$ - $\alpha$-L-arabinosyl transferases, and that BAHD genes encode feruloyl-arabinoxylan transferases. This group recently provided additional and compelling evidence for the participation of wheat GT61 genes, which they designated TaXAT, as xylan $(1,3)-\alpha$-L-arabinosyl transferases (Anders et al., 2012). Zeng et al. (2010) used GT43-specific antibodies to co-immunoprecipitate a complex from wheat microsomes that contained GT43, GT47, and GT75 proteins. Analysis of the glucuronoarabinoxylan polymer synthesized by the complex suggested a regular structure containing Xyl, Ara, and GluA in a ratio of 45:12:1. The authors suggested that this may represent a core complex in the biosynthetic process of xylans but to date we have no definitive evidence for the involvement of specific genes or proteins in the synthesis of the backbone or in the addition of certain substituents. Mortimer et al. (2010) reported that the products of two GT8 genes mediate the addition of GluA and 4-O-methylglucuronic acid residues to the heteroxylan of Arabidopsis. Double mutant plants for these genes, gux1gux2 contain xylan that is almost completely unsubstituted, but still contain wild type amounts of the xylan backbone. This indicates that the synthesis of the backbone and its substitution can be uncoupled; a somewhat surprising observation when the behavior of such an unsubstituted and hence probably insoluble polysaccharide in an aqueous environment is considered.

In another monocot/dicot difference, the heteroxylan from Arabidopsis contains a reducing terminal oligosaccharide consisting of $4-\beta$-D-Xylp- $(1,4)-\beta$-D-Xyl $p-(1,3)-\alpha$-L-Rhap- $(1,2)-\alpha-\mathrm{D}$ GalpA-(1,4)-D-Xyl $p$ (Pena et al., 2007). So far there is no evidence that such an oligosaccharide is present in arabinoxylans from the grasses (York and O'Neill, 2008).

\section{CHALLENGE II: DEFINING THE MECHANISM OF $(1,3 ; 1,4)-\beta$-GLUCAN SYNTHESIS AND ASSEMBLY}

As outlined in the sections above, the $(1,3 ; 1,4)-\beta$-glucans of the grasses can be considered a co-polymer consisting mainly of cellotriosyl (DP3) and cellotetraosyl (DP4) residues linked by single $(1,3)-\beta$-glycosidic linkages. The current challenge is therefore to 
devise a cellular and enzymic mechanism that would accommodate the random and non-random features described above, and then to come up with experimental evidence to prove the proposed mechanism.

At the moment it is not agreed exactly where the $(1,3 ; 1,4)$ $\beta$-glucans are synthesized in the cell. Wilson et al. (2006) used immunocytochemistry with specific monoclonal antibodies that detect adjacent $(1,3)$ - and $(1,4)-\beta$-glucosyl residues in polysaccharides (Meikle et al., 1994) to locate $(1,3 ; 1,4)-\beta$-glucans in several barley tissues. The polysaccharide could be clearly seen in the cell walls, but they could never detect any $(1,3 ; 1,4)-\beta$-glucan inside the cell, despite the long-held belief that the polysaccharide is synthesized in the Golgi apparatus. The same patterns were seen in barley leaves, coleoptiles, suspension-cultured cells, and in the developing grain. In contrast, Carpita and McCann (2010) reported that $(1,3 ; 1,4)-\beta$-glucan was indeed present in the Golgi of developing maize coleoptiles, using the same antibody. The situation is not helped by the paucity of information regarding the location of the $(1,3 ; 1,4)-\beta$-glucan synthase proteins themselves. Doblin et al. (2009) used an anti-HA antibody to show that the tagged barley CSLH protein, which is known to mediate $(1,3 ; 1,4)$ - $\beta$-glucan synthesis, was located in ER- and Golgi-derived vesicles in transgenic Arabidopsis tissues.

So, what possible mechanism would allow the HvCslH protein to be located in Golgi vesicles whilst the polysaccharide itself is only found in the wall? Doblin et al. (2009) and Burton et al. (2010) proposed that $(1,3 ; 1,4)$ - $\beta$-glucan synthesis might be a twophase process, through which the CslF and/or CslH enzymes of the Golgi synthesize a population of oligosaccharides with only $(1,4)$ $\beta$-linkages, that is cellodextrins. They envisaged that these would be predominantly cellotriose and cellotetraose, but could include longer cellodextrins of up to 10-12 residues in low abundance, and that they would be covalently attached to a lipid or protein molecule in the Golgi membrane. The nature of the putative lipid or protein carriers is unknown, although Fujino and Ohnishi (1979) reported the presence of cellotriosyl sitosterol and other glycosylated sitosterol, campesterol and stigmasterol derivatives in rice bran. Some interest was subsequently shown in these glycolipids as possible intermediates in cellulose synthesis (Delmer, 1999) but their role in polysaccharide synthesis in plants has never been satisfactorily demonstrated.

In the absence of any $(1,3)$ - $\beta$-linkages, these putative carrierlinked oligosaccharides would not be recognized by the $(1,3 ; 1,4)$ $\beta$-glucan antibody. Following transport of the cellodextrins to the plasma membrane, it is proposed that they would be polymerized through (1,3)- $\beta$-linkages either in the plasma membrane or in the periplasmic space and would be released into the wall as $(1,3 ; 1,4)$ $\beta$-glucans that would then be recognized by the antibody. The proposal depends on the presence of a population of $(1,4)-\beta$-linked oligoglucosides of different lengths and the random selection of these by the putative transferase enzyme during their polymerization; this would explain the random distribution of the DP3 and DP4 units in the final polysaccharide. Candidate transferase enzymes include members of the large family of xyloglucan endotransglycosidases (XETs) or GT2 callose synthases located in the plasma membrane, both of which are abundant in the grasses. An alternative possibility is that unidentified GT enzymes synthesize the $(1,4)-\beta$-linked oligoglucosides in the Golgi and that these are polymerized to form the final $(1,3 ; 1,4)-\beta$-glucan polysaccharide by Csl enzymes at the plasma membrane.

For those proteins known to be located in the Golgi, there is also the matter of orientation in the membrane that will have a fundamental impact on the way in which resultant polysaccharides are synthesized. Davis et al. (2010) used protease protection experiments to demonstrate that in Arabidopsis an odd number of transmembrane domains (five) dictates that CslA9 faces the Golgi lumen whilst CslC4, with an even number, faces the cytosol. We currently have no information about the topology of the CslF and CslH proteins involved in $(1,3 ; 1,4)-\beta$-glucan synthesis, whichever membranes they are embedded in, just as we have little information about the role of different members of the gene families, with seven or more $C s l F$ representatives and one to three $C s l H$ genes in various cereals. It is becoming clear that the CslF6 gene is more important than other members of the same family, based on observations that loss of CslF6 activity dramatically reduces the amount of $(1,3 ; 1,4)-\beta$-glucan in all barley tissues in the $b g l$ mutant (Taketa et al., 2012) and overexpression of the HvCslF6 gene had a significant effect on barley leaf and grain $(1,3 ; 1,4)-\beta$-glucan content and structure (Table 1; Burton et al., 2011). However, overexpression of $H v C s l F 4$ also influenced $(1,3 ; 1,4)-\beta$-glucan properties (Table 1; Burton et al., 2011) and the establishment of the mechanistic role of each of these proteins, be it in a complex or solo, will be a major step forward in our understanding of the synthesis of what appears to be one of the less complex polysaccharides in plant cell walls.

\section{CHALLENGE III: ASSIGNING A DEFINITIVE FUNCTION TO $(1,3 ; 1,4)-\beta$-GLUCANS IN THE GRASSES}

Intrinsic $(1,3 ; 1,4)-\beta$-glucan variability in the cereals and grasses present a number of interpretative challenges with respect to the contention that $(1,3 ; 1,4)-\beta$-glucans are structural components of cell walls. Despite the high concentrations of $(1,3 ; 1,4)-\beta$-glucans in the grains of some grasses, this is not so for all grasses (Table 1) whilst they are found at low levels in vegetative tissues. The conclusion from this variability is that the $(1,3 ; 1,4)-\beta$-glucans are not essential for cell wall integrity but they may impact wall function such as in rapidly expanding tissues exemplified by oat or rice coleoptiles (Hoson and Nevins, 1989; Chen et al., 1999). On the other hand, it is also clear that walls can accommodate very high levels of $(1,3 ; 1,4)$ - $\beta$-glucans, as seen in the starchy endosperm of Brachypodium grain (Guillon et al., 2012; Table 1). Overexpression of the HvCslF6 gene in transgenic barley resulted in increases of $40 \%$ or more in $(1,3 ; 1,4)-\beta$-glucan content of transgenic grain with even larger increases in vegetative tissues (Burton et al., 2011). In contrast, we have been singularly unsuccessful in our attempts to either increase or decrease cellulose content in barley plants transformed with CesA genes (our unpublished data).

Roulin et al. (2002) suggested that the $(1,3 ; 1,4)-\beta$-glucans in young barley leaves might function primarily as a secondary store of glucose that is mobilized during sugar depletion in the plant, based on their observations that when the seedlings were placed in the dark, $(1,3 ; 1,4)-\beta$-glucan levels dropped and the $(1,3 ; 1,4)-\beta$-glucan endo- and exohydrolases increased. A storage 
function for $(1,3 ; 1,4)-\beta$-glucans would be consistent with their high levels in Brachypodium grain, which contains $42 \%$ (w/w) $(1,3 ; 1,4)-\beta$-glucan and $6 \%(\mathrm{w} / \mathrm{w})$ starch (Guillon et al., 2012). These values can be compared with the barley grain, which typically contains about $4 \%(\mathrm{w} / \mathrm{w})(1,3 ; 1,4)-\beta$-glucan and $65 \%$ (w/w) starch (Trafford and Fincher, 2012). Overexpression of the barley CslF6 gene behind a grain-specific promoter resulted in both increases in $(1,3 ; 1,4)$ - $\beta$-glucan content and a concomitant decrease in starch content in the grain of transgenic lines (Burton et al., 2011).

One can certainly rationalize the potential function of $(1,3 ; 1,4)$ $\beta$-glucans as a store of metabolizable glucose in terms of the comparative enzymology of starch and $(1,3 ; 1,4)$ - $\beta$-glucan biosynthesis and mobilization. A single CslF or CslH enzyme is required for $(1,3 ; 1,4)-\beta$-glucan synthesis in plants (Burton et al., 2006; Doblin etal., 2009), while multiple enzymes are needed for the synthesis of amylose and amylopectin in starch granules, where there is the added complication of moving enzymes to the plastid. Similarly, $(1,3 ; 1,4)$ - $\beta$-glucans can be hydrolyzed to glucose through the action of just two enzymes, namely a $(1,3 ; 1,4)-\beta$ glucan endohydrolase and a $\beta$-glucan glucohydrolase (Hrmova and Fincher, 2001), while starch degradation again requires a battery of hydrolytic enzymes for its complete conversion to glucose. Thus, the enzymology and cell biology of $(1,3 ; 1,4)-\beta$-glucan synthesis and hydrolysis would appear to be simpler than for starch, although enzyme kinetics will obviously be an important factor and we cannot conclude at this stage whether or not $(1,3 ; 1,4)$ - $\beta$-glucans really function in the cellular energy balance in the grasses.

\section{REFERENCES}

Anders, N., Wilkinson, M. D., Lovegrove, A., Freeman, J., Tryfona, T., Pellny, T. K., Weimar, T., Mortimer, J. C., Stott, K., Baker, J. M., DefoinPlatel, M., Shewry, P. R., Dupree, P., and Mitchell, R. A. (2012). Glycosyl transferases in family 61 mediate arabinofuranosyl transfer onto xylan in grasses. Proc. Natl. Acad. Sci. U.S.A. 109, 989-993.

Bacic, A., and Stone, B. (1981). Chemistry and organization of aleurone cell wall components from wheat and barley. Funct. Plant Biol. 8, 475-495.

Brown, D. M., Goubet, F., Wong, V. W., Goodacre, R., Stephens, E., Dupree, P., and Turner, S. R. (2007). Comparison of five xylan synthesis mutants reveals new insight into the mechanisms of xylan synthesis. Plant J. 52, 1154-1168.

Brown, D. M., Zhang, Z., Stephens, E., Dupree, P., and Turner, S. R. (2009). Characterization of IRX10 and IRX10-like reveals an essential role in glucuronoxylan biosynthesis in Arabidopsis. Plant J. 57, 732-746.

Burton, R. A., Collins, H. M., Kibble, N. A. J., Smith, J. A., Shirley, N. J., Jobling, S. A., Henderson,

\section{CONCLUDING REMARKS}

In addressing the current challenges in plant cell wall biology, it must be acknowledged that some of these are likely to be met in a short time frame, especially with the interest of the biofuels industries and human health considerations driving our understanding forward at a very rapid rate. In this brief review we have highlighted areas where we believe there are fascinating discoveries to be made in the immediate future. We understand that the cellular and molecular mechanisms proposed for the assembly of $(1,3 ; 1,4)$ - $\beta$-glucans, which might also apply to other non-cellulosic wall polysaccharides, challenge the long-held views of how these polysaccharides are synthesized and where this process occurs in the cell. Just as challenging is to provide experimental evidence to support the proposed two-phase biosynthetic mechanism. Emerging evidence that hydrolytic enzymes somehow participate in wall polysaccharide synthesis is also an area that needs to be pursued. But as York and O'Neill (2008) commented, "our current rudimentary knowledge of the mechanisms of plant polysaccharide biosynthesis necessarily limits us to informed and modest conjecture." We now have a number of new but testable hypotheses for the enzymic mechanism and cell biology of cell wall polysaccharide assembly in plants, and the answers to these questions will undoubtedly generate new challenges in the future.

\section{ACKNOWLEDGMENTS}

We wish to acknowledge the enthusiasm and dedication of colleagues who have worked with us on the cell walls of the grasses over many years. We are grateful for the long-term and generous support of the Australian Research Council.

Collins, H. M., Burton, R. A., Topping, D. L., Liao, M. L., Bacic, A. and Fincher, G. B. (2010). Variability in fine structures of noncellulosic cell wall polysaccharides from cereal grains: potential importance in human health and nutrition. Cereal Chem. 87, 272-282.

Davis, J., Brandizzi, F., Liepman, A. H., and Keegstra, K. (2010). Arabidopsis mannan synthase CSLA9 and glucan synthase CSLC4 have opposite orientations in the Golgi membrane. Plant J. 64, 1028-1037.

Delmer, D. P. (1999). CELLULOSE BIOSYNTHESIS: exciting times for a difficult field of study. Annu. Rev. Plant Physiol. Plant Mol. Biol. 50, 245-276.

Doblin, M. S., Pettolino, F. A., Wilson, S. M., Campbell, R., Burton, R. A., Fincher, G. B., Newbigin, E., and Bacic, A. (2009). A barley cellulose synthase-like CSLH gene mediates $(1,3 ; 1,4)$-beta-D-glucan synthesis in transgenic Arabidopsis. Proc. Natl. Acad. Sci. U.S.A. 106, 59966001.

Ebringerová, A. (2005). Structural diversity and application potential of hemicelluloses. Macromol. Symp. 232, 1-12.
Fincher, G. B. (1975). Morphology and chemical composition of barley endosperm cell-walls. J. Inst. Brew. 81, 116-122.

Fincher, G. B., and Stone, B. A. (2004). Chemistry of Nonstarch Polysaccharides. Oxford: Elsevier.

Fujino, Y., and Ohnishi, M. (1979). Isolation and structure of diglycosylsterols and triglycosylsterols in rice bran. Biochim. Biophys. Acta 574, 94-102.

Gibeaut, D. M., and Carpita, N. C. (1993). Synthesis of $(1 \rightarrow 3)$, $(1 \rightarrow 4)$-beta-D-glucan in the Golgi apparatus of maize coleoptiles. Proc Natl. Acad. Sci. U.S.A. 90, 3850-3854.

Guillon, F., Larre, C., Petipas, F., Berger, A., Moussawi, J., Rogniaux, H., Santoni, A., Saulnier, L., Jamme, F., Miquel, M., Lepiniec, L., and Dubreucq, B. (2012). A comprehensive overview of grain development in Brachypodium distachyon variety Bd21. J. Exp. Bot. 63, 739-755.

Hoson, T., and Nevins, D. (1989). $\beta$ D-Glucan antibodies inhibit auxininduced cell elongation and changes in cell wall of Zea coleoptile segments. Plant Physiol. 90, 1353-1358.

Hrmova, M., and Fincher, G. B. (2001). Structure-function relationships of 
beta-D-glucan endo- and exohydrolases from higher plants. Plant Mol. Biol. 47, 73-91.

Izydorczyk, M. S., and Biliaderis, C. G. (1994). Studies on the structure of wheat endosperm arabinoxylans. Carbohydr. Polym. 24, 11.

Jacobs, D. R., and Gallaher, D. D. (2004). Whole grain intake and cardiovascular disease: a review. Curr. Atheroscler. Rep. 6, 415-423.

Jemal, A., Ward, E., Hao, Y., and Thun, M. (2005). Trends in the leading causes of death in the United States, 1970-2002. JAMA 294, 1255-1259.

Lazaridou, A., and Biliaderis, C. G. (2004). Cryogelation of cereal $\beta$ glucans: structure and molecular size effects. Food Hydrocoll. 18, 933-947.

McLaren, J. S. (2005). Crop biotechnology provides an opportunity to develop a sustainable future. Trends Biotechnol. 23, 339-342.

Meikle, P. J., Hoogenraad, N. J., Bonig, I., Clarke, A. E., and Stone, B. A. (1994). A (1 $\rightarrow 3,1 \rightarrow 4)$ $\beta$-glucan-specific monoclonal antibody and its use in the quantitation and immunocytochemical location of $(1 \rightarrow 3,1 \rightarrow 4)$ - $\beta$-glucans. Plant J. 5, 1-9.

Mitchell, R. A. C., Dupree, P., and Shewry, P. R. (2007). A novel bioinformatics approach identifies candidate genes for the synthesis and feruloylation of arabinoxylan. Plant Physiol. 144, 43-53.

Mortimer, J. C., Miles, G. P., Brown, D. M., Zhang, Z., Segura, M. P., Weimar, T., Yu, X., Seffen, K. A., Stephens, E., Turner, S. R., and Dupree, P. (2010). Absence of branches from xylan in Arabidopsis gux mutants reveals potential for simplification of lignocellulosic biomass. Proc. Natl. Acad. Sci. U.S.A. 107, 17409-17414.
Oikawa, A., Joshi, H. J., Rennie, E. A., Ebert, B., Manisseri, C., Heazlewood, J. L., and Scheller, H. V. (2010). An integrative approach to the identification of Arabidopsis and rice genes involved in xylan and secondary wall development. PLoS ONE 5, e15481. doi: 10.1371/journal.pone. 0015481

Pellny, T. K., Lovegrove, A., Freeman, J., Tosi, P., Love, C. G., Knox, J. P., Shewry, P. R., and Mitchell, R. A. (2012). Cell walls of developing wheat starchy endosperm: comparison of composition and RNA-Seq transcriptome. Plant Physiol. 158, 612-627.

Pena, M. J., Zhong, R., Zhou, G.-K., Richardson, E. A., O’Neill, M. A., Darvill, A. G., York, W. S., and Ye, Z.-H. (2007). Arabidopsis irregular xylem 8 and irregular xylem9: implications for the complexity of glucuronoxylan biosynthesis. Plant Cell 19, 549-563.

Persson, S., Caffall, K. H., Freshour, G., Hilley, M. T., Bauer, S., Poindexter, P., Hahn, M. G., Mohnen, D., and Somerville, C. (2007). The Arabidopsis irregular xylem8 mutant is deficient in glucuronoxylan and homogalacturonan, which are essential for secondary cell wall integrity. Plant Cell 19, 237-255.

Pettolino, F., Sasaki, I., Turbic, A., Wilson, S. M., Bacic, A., Hrmova, M., and Fincher, G. B. (2009). Hyphal cell walls from the plant pathogen Rhynchosporium secalis contain $(1,3$; 1,6)-beta-D-glucans, galacto- and rhamnomannans, $(1,3 ; 1,4)$-beta-Dglucans and chitin. FEBS J. 276, 3698-3709.

Roulin, S., Buchala, A. J., and Fincher, G. B. (2002). Induction of (1-3, $1-4)-\beta$-D-glucan hydrolases in leaves of dark-incubated barley seedlings. Planta 215, 51-59.

Staudte, R., Woodward, J., Fincher, G., and Stone, B. (1983). Water-soluble $(1,3 ; 1,4)$-beta-D-glucans in barley (Hordeum vulgare) endosperm. III. Distribution of celltriosyl and cellotetraosyl residues. Carbohydr. Polym. 3 , 299-312.

Taketa, S., Yuo, T., Tonooka, T., Tsumuraya, Y., Inagaki, Y., Haruyama, N., Larroque, O., and Jobling, S. A. (2012). Functional characterization of barley betaglucanless mutants demonstrates a unique role for CslF6 in $(1,3 ; 1,4)$-beta-D-glucan biosynthesis. J. Exp. Bot. 63, 381-392.

Topping, D. (2007). Cereal complex carbohydrates and their contribution to human health. J. Cereal Sci. 46, 220-229.

Trafford, K., and Fincher, G. B. (2012). "Barley grain carbohydrates: starch and cell walls," in Barley: Chemistry and Technology, ed. P. R. Shewry (Wallingford: CAB International) (in press).

Trethewey, J. A. K., Campbell, L. M., and Harris, P. J. (2005). (1,3;1,4)Beta-D-glucans in the cell walls of the poales (sensu lato): an immunogold labeling study using a monoclonal antibody. Am. J. Bot. 92, 16601674.

Wilson, S. M., Burton, R. A., Doblin, M. S., Stone, B. A., Newbigin, E. J., Fincher, G. B., and Bacic, A. (2006). Temporal and spatial appearance of wall polysaccharides during cellularization of barley (Hordeum vulgare) endosperm. Planta 224 655-667.

Woodward, J. R., Fincher, G. B., and Stone, B. (1983). Water-soluble $(1 \rightarrow 3,1 \rightarrow 4)-\beta$-D-glucans from barley (Hordeum vulgare) endosperm. II.
Fine structure. Carbohydr. Polym. 3, 207-225.

Xue, X., and Fry, S. C. (2012). Evolution of mixed-linkage $(1 \rightarrow 3,1 \rightarrow 4)$ beta-D-glucan (MLG) and xyloglucan in Equisetum (horsetails) and other monilophytes. Ann. Bot. 109 , 873-886.

York, W. S., and O’Neill, M. A. (2008). Biochemical control of xylan biosynthesis - which end is up? Curr. Opin. Plant Biol. 11, 258-265.

Zeng, W., Jiang, N., Nadella, R., Killen, T. L., Nadella, V., and Faik, A. (2010). A glucurono(arabino)xylan synthase complex from wheat contains members of the GT43, GT47, and GT75 families and functions cooperatively. Plant Physiol. 154, 78-97.

Conflict of Interest Statement: The authors declare that the research was conducted in the absence of any commercial or financial relationships that could be construed as a potential conflict of interest.

Received: 11 April 2012; accepted: 30 May 2012; published online: 15 June 2012. Citation: Burton RA and Fincher GB (2012) Current challenges in cell wall biology in the cereals and grasses. Front. Plant Sci. 3:130. doi: 10.3389/fpls.2012. 00130

This article was submitted to Frontiers in Plant Physiology, a specialty of Frontiers in Plant Science.

Copyright (c) 2012 Burton and Fincher. This is an open-access article distributed under the terms of the Creative Commons Attribution Non Commercial License, which permits non-commercial use, distribution, and reproduction in other forums, provided the original authors and source are credited. 